\title{
Schweizerische Neurologische Gesellschaft (SNG)
}

Swiss Neurological Society • Schweizerische Neurologische Gesellschaft Société Suisse de Neurologie • Società Svizzera di Neurologia
Die Neurologie ging am Ende des 19. Jahrhunderts aus der Psychiatrie («Nervenarzt»), Inneren Medizin und Neuroanatomie hervor und durchlief in der zweiten Hälfte des letzten Jahrhunderts einen tiefgreifenden Wandel. Als anfänglich rein diagnostische und bisweilen als «kontemplativ» belächelte Disziplin, die wegen der damals schon sichtbaren Komplexität aber auch bewundert und von Studenten gefürchtet wurde, hat sich die Neurologie zu einer Fachspezialität entwickelt, in der es praktisch keinen Zweig mehr ohne relevante therapeutische Optionen gibt und die auch in der Notfallmedizin eine zunehmend grössere Rolle spielt. Damit hat freilich die Komplexität noch weiter zugenommen, und der zwangsläufige, unumkehrbare Trend zur Subspezialisierung, bei der schon lange niemand mehr das ganze Fach überblickt, beginnt auch hier die Einheit des Faches zu bedrohen. Der Grund für diese Entwicklung liegt zum einen in der viel besseren Diagnostik und zum anderen im atemberaubenden Fortschritt der Grundlagenwissenschaften im Neurobereich, der das pathophysiologische Verständnis der neurologischen Krankheiten entscheidend verbessert hat.

Die Schweizerische Neurologische Gesellschaft feiert dieses Jahr ihr 100-Jahr-Jubiläum. Sie wurde am 15. November 1908 in Olten mit den Zielen der Förderung der Neurologie als Wissenschaft und der Pflege enger Beziehungen mit den Grenzgebieten, aber auch der Mitglieder untereinander und zur Förderung/Vertretung praktischer Interessen der Neurologie gegründet. Hinzugekommen ist hier im speziellen die Organisation und Durchführung wissenschaftlicher Tagungen, die Wahrung der Qualität ärztlichen Handelns, die Durchführung der FMH-Prüfungen. Sie vergibt keine Fähigkeitsausweise, dies wird von der assoziierten Schweizerischen Gesellschaft für klinische Neurophysiologie (SGKN) besorgt. Die Gesellschaft umfasst momentan 317 ordentliche Mitglieder.

\section{Kongresse/Sponsorenpool}

Die Gesellschaft organisiert zwei Kongresse im Jahr, wobei regelmässig kleinere, inhaltlich benachbarte Gesellschaften zur Förderung des Gedankenaustausches zwischen den Disziplinen dazu eingeladen werden. Als wegweisend herausgestellt hat sich die Erschaffung des sogenannten Sponsorenpools: Hier sind die involvierten Firmen eingeladen, einen Sponsorenbeitrag nicht personenbezogen, sondern in einen Pool einfliessen zu lassen, der es dann erlaubt, den Mitgliedern gemeinsam einen internationalen Kongressbesuch mitzufinanzieren.

\section{Neurology.ch}

Im Sinne des Wissenstransfers gibt die SNG sechsmal im Jahr ein Faltblatt zuhanden der internistischen und allgemeinmedizinischen Ärztinnen und Ärzte heraus, das in einer kurzen Übersicht neue Entwicklungen in der Neurologie beschreibt.

\section{Orphan-Drug-Probleme}

Ein Hauptproblem der Neurologie stellt die Tatsache dar, dass die Behandlung zahlreicher Erkrankungen sehr kostspielig ist (Immuntherapien, Parkinson-Behandlung, Epilepsiebehandlung). Ein relevanteres Problem zudem sind die zahlreichen seltenen Krankheiten, die zwar theoretisch behandelbar wären, wogegen aber entweder keine Medikamente existieren oder bei denen keine offizielle Indikation für ein vorhandenes Medikament (anderer Hauptindikation) bestehen. Die Pharmaindustrie hat kaum Interesse, entsprechende Medikamente für einen sehr kleinen Absatzmarkt zu entwickeln bzw. im Hinblick auf die neurologische Indikation zu prüfen. Im Falle fehlender Indikation werden die Medikamentenkosten von der Krankenkasse nicht übernommen. Das gilt z.B. für mehrere Immunsuppressiva.

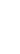

